\title{
HURST INDEX OF FUNCTIONS \\ OF LONG-RANGE-DEPENDENT MARKOV CHAINS
}

\author{
BARLAS OĞUZ*** AND \\ VENKAT ANANTHARAM,* University of California, Berkeley
}

\begin{abstract}
A positive recurrent, aperiodic Markov chain is said to be long-range dependent (LRD) when the indicator function of a particular state is LRD. This happens if and only if the return time distribution for that state has infinite variance. We investigate the question of whether other instantaneous functions of the Markov chain also inherit this property. We provide conditions under which the function has the same degree of long-range dependence as the chain itself. We illustrate our results through three examples in diverse fields: queueing networks, source compression, and finance.
\end{abstract}

Keywords: Markov chain; long-range dependence; Hurst index

2010 Mathematics Subject Classification: Primary 60J10

Secondary 68M20; 68P30; 91G70

\section{Introduction}

A stationary random process $\left(X_{n}\right)$ with $\mathrm{E}\left[X_{n}^{2}\right]<\infty$ is said to be long-range dependent (LRD) if

$$
\limsup _{n \rightarrow \infty} \sum_{r=1}^{n} \operatorname{cov}\left(X_{0}, X_{r}\right)=\infty .
$$

The degree of long-range dependence is measured by the Hurst index $H\left(\frac{1}{2} \leq H \leq 1\right)$ :

$$
H:=\inf \left\{h: \limsup _{n \rightarrow \infty} \frac{\sum_{r=1}^{n} \operatorname{cov}\left(X_{0}, X_{r}\right)}{n^{2 h-1}}<\infty\right\} .
$$

Equivalently, we can write

$$
H:=\inf \left\{h: \limsup _{n \rightarrow \infty} \frac{\operatorname{var}\left(\sum_{i=1}^{n} X_{i}\right)}{n^{2 h}}<\infty\right\} .
$$

Take $\left(M_{n}\right)$, a positive recurrent, aperiodic, discrete-time, countable state Markov chain with state space $\mathbb{N}$, where $\mathbb{N}$ denotes the set of natural numbers. The chain is in stationarity with stationary distribution $\pi$. The indicator function $1\left(M_{n}=i\right)$ of state $i$ of this chain is LRD if and only if indicator functions of every state are LRD [4]. When this is true, $\left(M_{n}\right)$ is said to be an LRD Markov chain. Moreover, the Hurst index of these functions is also a class property [4]. The common Hurst index $H$ is said to be the Hurst index of the chain.

Received 22 March 2011; revision received 28 November 2011.

* Postal address: Department of Electrical Engineering and Computer Sciences, University of California, Berkeley, CA 94720, USA.

** Email address: barlas@eecs.berkeley.edu 
In [4] it was proved that such a Markov chain is LRD if and only if the return time distribution of any state has infinite variance. It was also argued that finite weighted sums of indicator functions on this chain also inherit this property. It is natural to conjecture that this might be true for all functions of the chain. However, this conjecture is disproved, most easily by considering a constant function (see also the two counter examples in [4]). It is then of considerable interest to find which functions of an LRD Markov chain are also LRD.

Let $\varrho_{n}=\rho\left(M_{n}\right)$ be an $L_{2}$ function of $M_{n}$. In this paper, we provide conditions under which one can infer the long-range dependence of $\left(\varrho_{n}\right)$ from that of $\left(M_{n}\right)$.

Our main result, given in Section 3, provides a technical condition under which the rate of growth of $\sum_{r=1}^{n} \operatorname{cov}\left(X_{0}, X_{r}\right)$ is identical for $X_{n}=\varrho_{n}$ and $X_{n}=1\left(M_{n}=i\right)$. We set up the proof with a collection of lemmas presented in Section 8. For convenience, most of the notation is collected together in Section 2.

There are many interesting scenarios where the results of this paper might be useful. In the second half of the paper, we collect three such examples. In Section 4 we discuss a simple queueing network of two parallel queues. One queue is driven by an LRD process, whereas the other queue is driven by a short-range-dependent process. We model the inputs and queue lengths by countable state Markov chains, and show that, under longest queue first scheduling, both queues are LRD.

A particularly novel example is given in Section 5, where we re-prove a recent result in the source coding of LRD sequences [13]. We show that the code length process of any lossless encoder which is compressing an LRD renewal process must dominate an LRD process with the same Hurst index as the source process.

The last example concerns the long-range dependence in financial series. We discuss how our model can explain the LRD behavior observed in some instantaneous functions of the absolute returns of some asset.

\section{Notation and setup}

Let $\left(M_{n}\right)$ be a positive recurrent, discrete-time, countable state Markov chain with state space $\mathbb{N}$ and stationary distribution $\pi_{i}, i \in \mathbb{N}$. Most of the notation we use is borrowed from [5].

- $\rho: \mathbb{N} \rightarrow \mathbb{R}$ is such that $\sum_{i \in \mathbb{N}} \rho(i)^{2} \pi_{i}<\infty$.

- $\varrho_{n}:=\rho\left(M_{n}\right)$.

- $\mu:=\sum_{i} \rho(i) \pi_{i}$ is the mean of $\rho$.

- $p_{i j}^{(n)}:=\mathrm{P}\left(M_{n}=j \mid M_{0}=i\right), n \geq 0$, is the $n$-step transition probability from $i$ to $j$.

- ${ }_{k} p_{i j}^{(n)}:=\mathrm{P}\left(M_{n}=j ; M_{l} \neq k, 0<l<n \mid M_{0}=i\right), n>0$, is the $n$-step transition probability from $i$ to $j$ with taboo state $k$.

- ${ }_{k} p_{i j}^{*}:=\sum_{n=1 k}^{\infty} p_{i j}^{(n)}$.

- ${ }_{\mathscr{H}} p_{i j}^{(n)}:=\mathrm{P}\left(M_{n}=j ; M_{l} \notin \mathscr{H}, 0<l<n \mid M_{0}=i\right), n>0$, is the $n$-step transition probability from $i$ to $j$ with taboo set $\mathscr{H}$.

- ${ }_{\mathscr{H}} p_{i j}^{*}:=\sum_{n=1 \mathscr{H}}^{\infty} p_{i j}^{(n)}$.

- $f_{i j}^{(n)}:={ }_{j} p_{i j}^{(n)}, n>0$.

- $Q_{i j}^{(n)}:=\sum_{r=1}^{n}\left(p_{i j}^{(r)}-\pi_{j}\right), n>0$. 
- $R_{i j}^{(n)}:=\sum_{r=1}^{n} Q_{i j}^{(r)}, n>0$.

- $T_{j}:=\inf _{t}\left\{t>0: M_{t}=j\right\}$ is the first time to state $j$ at stationarity.

- $m_{i j}:=\mathrm{E}_{i}\left[T_{j}\right]$ is the mean time to state $j$ starting from $i$.

- $H:=\inf \left\{h: \lim \sup _{n \rightarrow \infty} \operatorname{var}\left(\sum_{i=1}^{n} \mathbf{1}\left(M_{i}=1\right)\right) / n^{2 h}<\infty\right\}$ is the Hurst index of $\left(M_{n}\right)$.

- $H_{\varrho}:=\inf \left\{h: \lim \sup _{n \rightarrow \infty} \operatorname{var}\left(\sum_{i=1}^{n} \varrho_{i}\right) / n^{2 h}<\infty\right\}$ is the Hurst index of $\left(\varrho_{n}\right)$.

To understand the results in the next section, it is useful to know the following properties.

Lemma 2.1. For an LRD Markov chain,

$$
\begin{gathered}
\lim _{n \rightarrow \infty} Q_{i j}^{(n)}=\infty, \\
\lim _{n \rightarrow \infty} \frac{R_{i j}^{(n)}}{n}=\infty, \\
\lim _{n \rightarrow \infty} \frac{Q_{i j}^{(n)} / \pi_{j}}{Q_{11}^{(n)} / \pi_{1}}=1 .
\end{gathered}
$$

Proof. Equation (2.3) is Equation 8 of [4]. Equation (2.1) follows from Equations 8 and 5 of [4]. Equation (2.2) follows from (2.1). This completes the proof.

We will assume henceforth that $n$ is large enough such that $Q_{11}^{(n)}, R_{11}^{(n)}>1$.

\section{Main results}

Theorem 3.1. Let

(i) $\lim _{n \rightarrow \infty} \frac{1}{Q_{11}^{(n)} / \pi_{1}} \sum_{r=1}^{n} \sum_{i, j} \pi_{i}(\rho(i)-c)(\rho(j)-c)_{\mathcal{H}} p_{i j}^{(r)}=0$

for some constant $c$ and nonempty, finite set $\mathcal{H}$, and

(ii) $\lim _{L \rightarrow \infty} \limsup _{n \rightarrow \infty} \frac{1}{Q_{11}^{(n)} / \pi_{1}} \sum_{r=1}^{n} \sum_{i, j} \pi_{i}|\rho(i) \rho(j)| 1(|\rho(i)|>L,|\rho(j)|>L)_{\mathcal{H}} p_{i j}^{(r)}=0$.

Then

$$
\lim _{n \rightarrow \infty} \frac{\operatorname{var}\left(\sum_{r=1}^{n} \varrho_{i}\right)}{R_{11}^{(n)} / \pi_{1}}=(\mu-c)^{2} .
$$

Moreover, if $c \neq \mu$ then $H_{\varrho}=H$.

Some remarks about Theorem 3.1(i) and (ii) are in order.

1. They fail to hold if $\lim _{i} \rho(i)$ exists and is not $c$. This shows that $\lim _{i} \rho(i)$ is the unique choice for $c$ in this case.

2. They will hold whenever $\lim _{i}(\rho(i)-c)=0$. Specifically, when $\rho(i)-c=0$ for $i$ greater than some value.

Both these remarks follow directly from Lemma 8.6, which is stated in Section 8. 
3. They are implied by the considerably stronger condition

$$
\frac{1}{Q_{11}^{(n)} / \pi_{1}} \sum_{r=1}^{n} \sum_{i, j} \pi_{i}|\rho(i)-c||\rho(j)-c|_{1} p_{i j}^{(r)} \rightarrow 0 .
$$

Theorem 3.1(ii) is trivially satisfied for bounded functions. When the $\varrho_{n}$ are not bounded, condition (ii) ensures that they can be truncated without affecting the long-range dependence discussions.

In light of remark 2, $c$ can be interpreted as a 'limiting mean', in a weak sense, of $\varrho$ as the return time to the compact set $\mathscr{H}$ becomes large. The deviance of $\varrho$ from its average behavior in this limiting regime, given by $\mu-c$, determines the limiting constant in the statement of Theorem 3.1. When $(\mu-c)^{2}=0$, the behavior of $\varrho$ is similar to its average behavior even when $M_{n}$ takes a long excursion before returning to $\mathscr{H}$. Therefore, the long-range dependence of $M$ might not exhibit itself in $\varrho$. In this case $\varrho$ might have a lower Hurst index, or even be short-range dependent. What happens exactly depends on the detailed structure of $M$ and $\rho$, and cannot be captured by our formulation which only investigates the asymptotics at the scale of the Hurst index of $M$. In this regard, $(\mu-c)^{2}>0$ is necessary for $\varrho$ to be LRD at the same scale as $M$, and examples can easily be constructed to show that the $\varrho$ that fail this condition fail to be LRD to the same degree. We give one such nonexample in Section 7.

The following theorem extends the usefulness of the preceding theorem considerably. It describes the case when the state space of the Markov chain is divided into a finite number of subsets, with communication between the sets happening almost only through a finite set of states $\mathscr{H}$. The canonical example for such a structure would be the Markov chain representation of a semi-Markov process given by the pair $(S, T)$, where $S$ is described by a finite-state Markov chain and $T$ is the time since the last transition, having an arbitrary distribution with $\mathrm{E}[T]<\infty$. In this case, the state space would be divided into sets $\{S=k\}$, and transition between sets is only possible by visiting $(S, 0)$.

Theorem 3.2. Let $\left\{\mathcal{A}_{k}\right\}, 1 \leq k \leq K$, be a finite partition of the state space $\mathbb{N}$. Let $\mathscr{H}$ be a nonempty finite set, and let

(i) $\lim _{n \rightarrow \infty} \frac{1}{Q_{11}^{(n)} / \pi_{1}} \sum_{r=1}^{n} \sum_{i \in \mathcal{A}_{k}, j \in \mathcal{A}_{l}} \pi_{i}|\rho(i)-\mu||\rho(j)-\mu|_{\mathcal{H}} p_{i j}^{(r)}=0$ for all $k \neq l$.

Also, suppose that

$$
\pi_{\mathcal{A}_{k}}^{\infty}:=\lim _{n \rightarrow \infty} \frac{\sum_{i, j \in \mathcal{A}_{k}} \pi_{i} \sum_{r=11}^{n} p_{i j}^{(r)}}{\sum_{i, j} \pi_{i} \sum_{r=11}^{n} p_{i j}^{(r)}}
$$

exists for all $k$. Let there exist constants $c_{k}, 1 \leq k \leq K$, such that, for all $k$,

(ii) $\lim _{n \rightarrow \infty} \frac{1}{Q_{11}^{(n)} / \pi_{1}} \sum_{r=1}^{n} \sum_{i, j \in \mathcal{A}_{k}} \pi_{i}\left(\rho(i)-c_{k}\right)\left(\rho(j)-c_{k}\right)_{\mathcal{H}} p_{i j}^{(r)}=0$

and

(iii) $\lim _{L \rightarrow \infty} \limsup _{n \rightarrow \infty} \frac{1}{Q_{11}^{(n)} / \pi_{1}} \sum_{r=1}^{n} \sum_{i, j \in \mathcal{A}_{k}} \pi_{i}|\rho(i) \rho(j)| 1(|\rho(i)|>L,|\rho(j)|>L)_{\mathscr{H}} p_{i j}^{(r)}=0$. 
Then

$$
\lim _{n \rightarrow \infty} \frac{\operatorname{var}\left(\sum_{r=1}^{n} \varrho_{i}\right)}{R_{11}^{(n)} / \pi_{1}}=\sum_{k=1}^{K} \pi_{\mathcal{A}_{k}}^{\infty}\left(\mu-c_{k}\right)^{2} .
$$

Moreover, if $\pi_{\mathcal{A}_{k}}^{\infty}\left(c_{k}-\mu\right) \neq 0$ for some $k$ then $H_{\varrho}=H$.

Note that if $c_{k}=c_{l}$ for a pair of subsets $\mathcal{A}_{k}, \mathcal{A}_{l}$ then $(i)$ is not needed for this particular pair.

Theorem 3.2(ii) defines a 'limiting mean' $c_{k}$ for $\varrho$ in each set $\mathcal{A}_{k}$, as Theorem 3.1(i) did. Condition (iii) is the analogue of Theorem 3.1(ii). Condition (i) ensures that transition events across different sets $\mathcal{A}_{k}$ without visiting $\mathscr{H}$ can be ignored. Here $\pi_{\mathcal{A}_{k}}^{\infty}$ can be regarded as the limiting probability of $\mathscr{A}_{k}$ as the return time back to $\mathscr{H}$ becomes large.

As before, $\pi_{\mathcal{A}_{k}}^{\infty}\left(c_{k}-\mu\right) \neq 0$ for at least one $k$ is necessary for the long-range dependence of $\varrho$ to be at the same scale as $M$.

We now illustrate the use of these tools with some examples. In the first example we use Theorem 3.1 directly, while in the last two examples we use Theorem 3.2.

\section{Example 1: longest queue first with mixed heavy- and light-tailed inputs}

This example replicates the conclusion in [12] that long-range dependence might spread under longest queue first (LQF) scheduling in a parallel queue setting, using a general technique based on Theorems 3.1 and 3.2.

There is a single server of rate $R \in \mathbb{N}$ with two parallel queues. The queues are fed by independent random processes, each modeled by a discrete-time, countable state Markov chain. As an example, we investigate the scenario where $X_{1}$ is independent and identically distributed (i.i.d.) with heavy-tailed $\left(\operatorname{var}\left(X_{1}\right)=\infty\right)$ arrival distribution on $\mathbb{N}$. Either $X_{2} \in \mathbb{N}$ is an i.i.d. process with light-tailed $\left(\operatorname{var}\left(X_{2}\right)<\infty\right)$ arrivals or $X_{2}$ is a finite-state, $\mathbb{N}$-valued Markov chain in stationarity. We assume that $\mathrm{E}\left[X_{1}(0)\right]+\mathrm{E}\left[X_{2}(0)\right]<R$.

Let $Q_{1}(n)$ and $Q_{2}(n)$ be the stationary queue lengths. We assume that the queue is work conserving, and moreover that the scheduling decision at time $n$ (number of packets to be served from each queue at time slot $n)$ is a function of $\left(Q_{1}(n), Q_{2}(n)\right)$, the queue sizes at time $n$. Given such a scheduling strategy, it is easily verified that $\left(X_{1}(n), X_{2}(n), Q_{1}(n), Q_{2}(n)\right)$ is a countable state Markov chain.

Lemma 4.1. $\left(X_{1}(n), X_{2}(n), Q_{1}(n), Q_{2}(n)\right)$ is positive recurrent.

Proof. The condition

$$
\mathrm{E}\left[X_{1}(0)\right]+E\left[X_{2}(0)\right]<R
$$

implies that the queue process $\left(Q_{1}(n), Q_{2}(n)\right)$ is positive recurrent. Pick $M_{1}>0$, and define the set $S_{1}=\left\{Q_{1}(n)+Q_{2}(n)<M_{1}\right\}$. The return times to this set have finite mean (say $\left.v\right)$. Also, define $S_{2}=\left\{X_{1}(n)+X_{2}(n)<M_{2}\right\}$ (or in the case where $X_{2}$ is a finite state chain, $S_{2}=\left\{X_{1}(n)<M_{2}\right\}$ ), where $M_{2}$ is large enough such that $S_{2}$ is nonempty. $S_{1} \cap S_{2}$ is a nonempty compact set. We claim that the return times to this set have a finite mean. Since $1_{n}\left(S_{2}\right)$ is i.i.d., there is a positive probability (say at least $p$ ) of visiting $S_{2}$ each time there is a visit to $S_{1}$ (independent of previous visits). It is easily seen that the mean return time to $S_{1} \cap S_{2}$ is at most $v / p$ (expectation of a sum of geometrically many i.i.d. variables). This completes the proof.

We will look at long-range dependence through the Hurst indices of the busy-idle processes of the queues. Let $\left(X_{1}, Q_{1}^{\prime}\right)$ be the Markov chain in which all the capacity is allocated to 
queue 1 . Denote by $1\left(Q_{1}^{\prime}(n)=0\right)$, the busy-idle process of this queue. We know that the busy periods of $Q_{1}^{\prime}$ have infinite variance (see, e.g. [3, Theorem 8.10.3]). Therefore, both the Markov chain $\left(X_{1}, Q_{1}^{\prime}\right)$ and the function $1\left(Q_{1}^{\prime}(n)=0\right)$ are LRD (see the introduction). The similarly defined $\left(X_{2}, Q_{2}^{\prime}\right)$ is a short-range-dependent chain.

Lemma 4.2. $\left(X_{1}(n), X_{2}(n), Q_{1}(n), Q_{2}(n)\right)$ is $L R D$.

Proof. Consider the chain $\left(X_{1}(n), Q_{1}^{\prime}(n), X_{2}(n), Q_{2}^{\prime}(n)\right)$. This chain is LRD because it is a combination of the two independent chains $\left(X_{1}, Q_{1}^{\prime}\right)$ and $\left(X_{2}, Q_{2}^{\prime}\right)$, one of which we assume to be LRD. Let $t_{1}$ be the return time to a nonempty compact set $S_{1}=\left\{X_{1}(n), Q_{1}(n), X_{2}(n)\right.$, $\left.Q_{2}(n)<M\right\}$. Similarly, let $t_{2}$ be the return time to the set $S_{2}=\left\{X_{1}(n), Q_{1}^{\prime}(n), X_{2}(n)\right.$, $\left.Q_{2}^{\prime}(n)<M\right\}$. Since $Q_{1}^{\prime}(n) \leq Q_{1}(n)$ and $Q_{2}^{\prime}(n) \leq Q_{2}(n), t_{1}$ stochastically dominates $t_{2}$, and therefore $\left(X_{1}(n), X_{2}(n), Q_{1}(n), Q_{2}(n)\right)$ is also LRD. This completes the proof.

The question we want to ask then is whether $1\left(Q_{2}(n)=0\right)$, the busy-idle process of the second queue (fed by short-range-dependent traffic), is also LRD.

Define $\varrho_{n}:=1\left(Q_{2}(n)=0\right)$ to be an $L_{2}$ function of the chain $\left(X_{1}(n), X_{2}(n), Q_{1}(n), Q_{2}(n)\right)$. Take $c=0$ in Theorem 3.1. Let $\mathscr{H}=\left\{X_{1}(n), X_{2}(n), Q_{1}(n), Q_{2}(n) \leq R\right\}$. Condition (ii) holds trivially for bounded functions. Thus, we are left with having to check the condition

$$
\lim _{n \rightarrow \infty} \frac{1}{Q_{11}^{(n)} / \pi_{1}} \sum_{\left\{i, j: Q_{2, j}=0, Q_{2, i}=0\right\}} \pi_{i} \sum_{r=1}^{n}{ }_{H} p_{i j}^{(r)}=0 .
$$

To see why this is true, note that $\sum_{\left\{i, j: Q_{2, j}=0, Q_{2, i}=0\right\}} \pi_{i} \sum_{r=1}^{\infty}{ }_{H} p_{i j}^{(r)}$ is bounded above by 1 plus the stationary time spent in the states $\left\{Q_{2}=0\right\}$ before the chain visits $\mathscr{H}$. Note that the length of an idle period for $Q_{2}$ has finite expectation. Also, note that if an idle period begins at time $n+1$, this implies, owing to the LQF policy, that $Q_{1}(n) \leq R, Q_{2}(n) \leq R$, $X_{1}(n) \leq R$, and $X_{2}(n) \leq R$. Thus, between successive idle periods of $Q_{2}$, the chain must visit $\mathscr{H}$. The stationary expected time spent in $\left\{Q_{2}=0\right\}$ without visiting $\mathscr{H}$ is therefore finite. Since $Q_{11}^{(n)} \rightarrow \infty$ (by (2.1)), the above limit holds. Using Theorem 3.1, we conclude that $1\left(Q_{2}(n)=0\right)$ has the same Hurst index as the chain $\left(X_{1}(n), X_{2}(n), Q_{1}(n), Q_{2}(n)\right)$.

The advantage of this approach is that in general the input processes need not be i.i.d. Dependencies can easily be modeled, as long as the sources can be represented as countable state Markov.

\section{Example 2: compressing an LRD renewal process}

In this section we provide an alternative proof for the result in [13].

Let $\left(X_{n}\right) \in\{0,1\}$ be a discrete, stationary, ergodic renewal process. Denote by $\tau_{1}$ and $\tau_{2}$ the times of the first two arrivals. Then we denote by $T \stackrel{\mathrm{D}}{=} \tau_{2}-\tau_{1}$ a random variable having the interarrival distribution. We assume that $\mathrm{E}[T]<\infty$ and $\mathrm{E}\left[T^{2}\right]=\infty$. As discussed in the introduction, this is equivalent to stating that the renewal process is LRD. (For instance, consider a Markov chain with return times to state 1 having the law of $T$. Then the renewal process can be defined as the indicator function of state 1.)

We begin by introducing the function

$$
\varrho_{n}\left(X_{-\infty}^{n}\right)=-\log \mathrm{P}\left(X_{n} \mid X_{-\infty}^{n-1}\right)
$$

which is of central importance to coding theory. The behavior of $\left(\varrho_{n}\right)$ restricts the minimum code length of lossless compression algorithms by the following lemma from [1], which is also proved in [10]. 
Lemma 5.1. (Barron's lemma.) Given $\{c(n), n \geq 1\}$, positive constants with $\sum_{n} 2^{-c(n)}<\infty$, we have

$$
L_{n}\left(X_{1}^{n}\right) \geq-\log \mathrm{P}\left(X_{1}^{n} \mid X_{-\infty}^{0}\right)-c(n) \quad \text { eventually, almost surely (a.s.). }
$$

Here $L_{n}\left(X_{1}^{n}\right)$ is the code length for the first $n$ symbols of the source for some lossless coding algorithm that produces bit strings. (That is, let $L_{n}\left(X_{1}^{n}\right)$ be the length of $\phi\left(X_{1}^{n}\right)$, where $\phi\left(x_{1}^{n}\right):\{0,1\}^{n} \rightarrow\{0,1\}^{*}$ is a one-to-one mapping.) $c(n)$ can be made logarithmic in $n$.

By the ergodic theorem, the limit of $(1 / n) \sum_{i=1}^{n} \varrho_{i}$ as $n \rightarrow \infty$ exists a.s. and equals $\eta:=$ $\mathrm{E}\left[-\log \mathrm{P}\left(X_{1} \mid X_{-\infty}^{0}\right)\right]$, i.e. the entropy rate of $\left(X_{n}\right)$. This implies the following well-known first-order converse source coding theorem for such sources.

Theorem 5.1. It holds that

$$
\lim \inf _{n} \frac{1}{n} L_{n}\left(X_{1}^{n}\right) \geq \eta \quad \text { a.s. }
$$

Lemma 5.1 is strong enough to permit second-order refinements to Theorem 5.1 once we know more about the process $\left(\varrho_{n}\right)$. For example, in [10], it was shown that, for certain shortrange-dependent classes of sources (e.g. finite-state Markov chains) and appropriate coding schemes (e.g. Lempel-Ziv coding), $\left(L_{n}-n \eta\right)$ satisfies a central limit theorem.

Here, we will prove a second-order converse source coding theorem, stating that the bit length process $\left(L_{n}\right)$ will eventually dominate an LRD process, the growth of whose variance is identical to that of $\left(X_{n}\right)$, so that, in particular, it has the same Hurst exponent as $\left(X_{n}\right)$. The proof relies on our general Theorem 3.2. This result provides partial theoretical justification to existing empirical work in the field of variable bit-rate (VBR) video traffic (see [2], [7], [8], and [14], among others). A conclusion resulting from this work is that long-range dependence is omnipresent in VBR video traffic, and persists across a wide variety of codecs. Combined with these observations, the result backs the intuition that, for many information sources, long-range dependence persists under compression.

Theorem 5.2. Let $\left(X_{n}\right)$ be an aperiodic, LRD, stationary, ergodic renewal process. Then there exists an LRD random process $\left(\gamma_{n}\right)$ such that

$$
L_{n}\left(X_{1}^{n}\right) \geq \gamma_{n} \text { eventually, a.s. }
$$

for all uniquely decodable source codes. Moreover, $\left(\gamma_{n}\right)$ has the same Hurst index as $\left(X_{n}\right)$.

Proof. This follows immediately from Barron's lemma once we show that the $\left(\varrho_{n}\right)$ are LRD with the same Hurst index as $\left(X_{n}\right)$. This will follow from Theorem 3.2 if we can set up $\left(\varrho_{n}\right)$ as a function of a Markov chain.

We construct the following Markov chain $\left(M_{n}\right)$ from the renewal process $\left(X_{n}\right)$ (see Figure 1):

- $M_{n} \in\{0,1,2,3, \ldots\}$,

- $\left\{M_{n}=0\right\}=\left\{X_{n-1}^{n}=11\right\}$,

- for $k \in\{1,2, \ldots\}$,

- $\left\{M_{n}=2 k-1\right\}=\left\{X_{n}=0\right.$ and $k$ zeros since last arrival $\}$,

- $\left\{M_{n}=2 k\right\}=\left\{X_{n}=1\right.$ and $k$ zeros since last arrival in $\left.X_{n}\right\}$. 


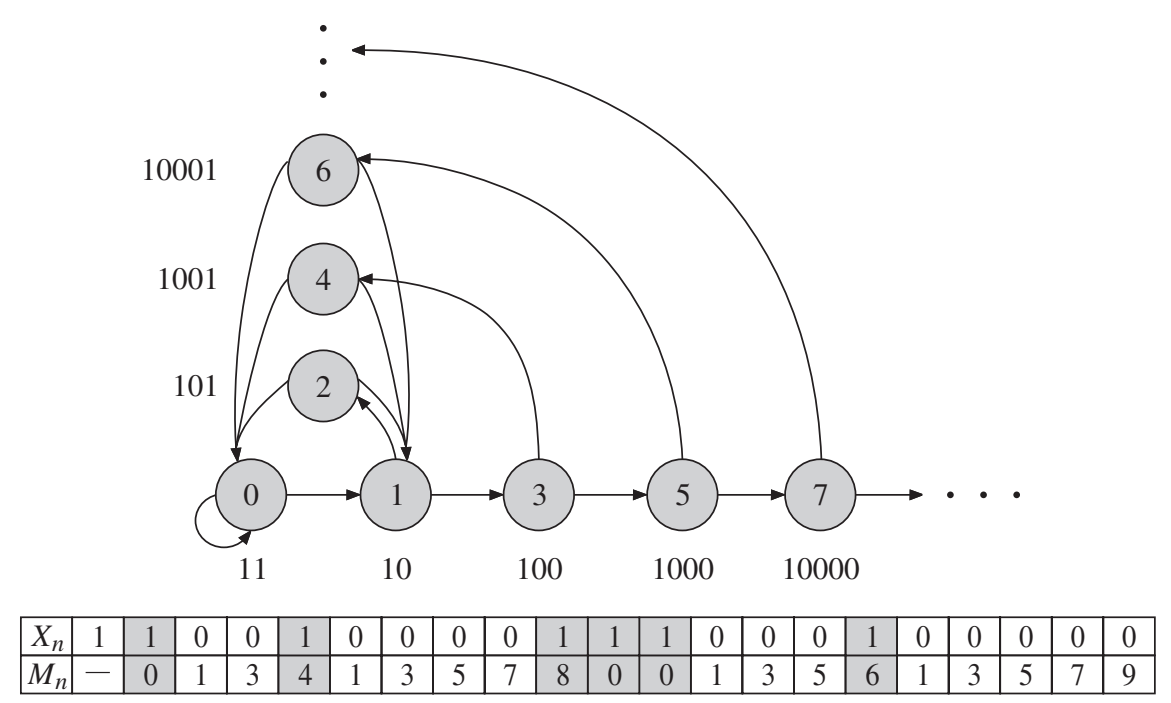

Figure 1: Construction of the Markov chain $\left(M_{n}\right)$, with an example sequence showing the correspondence with $X_{n}$.

Note that this Markov chain is equivalent to the characterization $\left(X_{n}, t_{n}\right)$ (where $t_{n}$ is the time since the last transition), only the states are numbered such that the state space is $\mathbb{N}$.

We establish some additional notation: let $\left(X_{n}\right)$ denote the stationary renewal process, whose interval arrival lengths have the law of $T+1 ; f_{T}(k):=\mathrm{P}(T=k) ; F_{T}(k):=\mathrm{P}(T \leq k)$; $\varrho_{n}\left(X_{-\infty}^{n}\right):=-\log \mathrm{P}\left(X_{n} \mid X_{-\infty}^{n-1}\right) ;$ and $\eta:=\mathrm{E}\left[\log \mathrm{P}\left(X_{1} \mid X_{-\infty}^{0}\right)\right]$.

We can easily check that $\varrho_{n}=\rho\left(M_{n}\right)$, with

- $\rho(0)=-\log f_{T}(0)$,

- $\rho(2 k-1)=-\log \mathrm{P}(T>k-1 \mid T \geq k-1)$,

- $\rho(2 k)=-\log \mathrm{P}(T=k \mid T \geq k)$.

Lemma 5.2. $\varrho_{n}$ is an $L_{2}$ function of $M_{n}$.

Proof. Let $\pi_{i}$ be the stationary distribution of $\left(M_{n}\right)$. Note that $\pi_{i}>0$ implies that $\rho(i)<\infty$. We want to prove that

$$
\sum \rho(i)^{2} \pi_{i}<\infty
$$

Note that $\pi_{2 k+1}=\pi_{2 k-1} \mathrm{P}(T>k \mid T \geq k)$ and $\pi_{2 k}=\pi_{2 k-1} \mathrm{P}(T=k \mid T \geq k)$ for $k=1,2, \ldots$ This gives

$$
\begin{aligned}
\sum \rho(i)^{2} \pi_{i}= & \pi_{0} \rho(0)^{2}+\pi_{1} \rho(1)^{2}+\sum_{k=1}^{\infty} \pi_{2 k-1} \mathrm{P}(T=k \mid T \geq k) \log ^{2} \mathrm{P}(T=k \mid T \geq k) \\
& +\sum_{k=1}^{\infty} \pi_{2 k-1} \mathrm{P}(T>k \mid T \geq k) \log ^{2} \mathrm{P}(T>k \mid T \geq k), \\
\pi_{0} \rho(0)^{2}= & \left(\sum_{k=1}^{\infty} \pi_{2 k}\right) f_{T}(0) \log ^{2} f_{T}(0),
\end{aligned}
$$


and

$$
\pi_{1} \rho(1)^{2}=\left(\sum_{k=1}^{\infty} \pi_{2 k}\right)\left(1-f_{T}(0)\right) \log ^{2}\left(1-f_{T}(0)\right) .
$$

Since the $p \log ^{2} p$ terms are bounded above by $1, \sum \rho(i)^{2} \pi_{i} \leq 4$. This completes the proof.

Now, to apply Theorem 3.2, we partition into three sets: $\mathcal{A}_{1}=\{i>0$ : even $i\}, \mathcal{A}_{2}=$ $\{0\} \cup\left\{\right.$ odd $\left.i: \rho(i) \leq-\log \left(1-\varepsilon_{i}\right)\right\}$, and $\mathscr{A}_{3}=\left\{\right.$ odd $\left.i: \rho(i)>-\log \left(1-\varepsilon_{i}\right)\right\}$. We will choose $\varepsilon_{i} \downarrow 0$ later. Take $c_{1}=c_{2}=c_{3}=0$ and $\mathscr{H}=1$ in Theorem 3.2. By the note at the end of the statement of Theorem 3.2, we do not need condition (i). We will check that conditions (ii) and (iii) hold for each of the sets.

When $i, j \in \mathcal{A}_{1}$, note that ${ }_{1} p_{i j}^{(r)}=0$, so both conditions hold automatically. For $i, j \in \mathcal{A}_{2}$, condition (ii) holds due to remark 2 because the limit of $\rho(i)$ as $i \rightarrow \infty$ is 0 , and condition (iii) holds because $\rho$ is bounded on this set. Thus, we focus on $i, j \in \mathcal{A}_{3}$. Define $\rho(i)=$ : $-\log \left(1-\tilde{\varepsilon}_{i}\right)$. Let subsequence $\left\{i_{k}\right\}=\mathcal{A}_{3}$. We have $\tilde{\varepsilon}_{i_{k}} \geq \varepsilon_{i_{k}}, \pi_{i_{k}} \leq \pi_{1} \prod_{l=1}^{k}\left(1-\tilde{\varepsilon}_{i_{k}}\right)$, and $\sum_{1}^{\infty} p_{i_{k} i_{j}}^{(r)}=\pi_{i_{j}} / \pi_{i_{k}}$. Thus,

$$
\begin{aligned}
& \sum_{i} \rho(i) \pi_{i} \sum_{j} \rho(j) \sum_{r=1}^{n}{ }_{1} p_{i j}^{(r)} \\
& \quad \leq \sum_{k} \prod_{l=1}^{k}\left(1-\tilde{\varepsilon}_{i_{l}}\right)\left(-\log \left(1-\tilde{\varepsilon}_{i_{k}}\right)\right) \sum_{j>k}-\log \left(1-\tilde{\varepsilon}_{i_{j}}\right) \prod_{l=k+1}^{j}\left(1-\tilde{\varepsilon}_{i_{l}}\right) \\
& \quad=\sum_{j} \sum_{k<j}\left(1-\tilde{\varepsilon}_{i_{k}}\right) \log \left(1-\tilde{\varepsilon}_{i_{k}}\right)\left(1-\tilde{\varepsilon}_{i_{j}}\right) \log \left(1-\tilde{\varepsilon}_{i_{j}}\right) \prod_{l=1, l \neq k, j}^{j}\left(1-\tilde{\varepsilon}_{i_{l}}\right) \\
& \quad<\sum_{j} j \prod_{l=3}^{j}\left(1-\tilde{\varepsilon}_{i_{l}}\right) .
\end{aligned}
$$

We can easily choose $\varepsilon_{i} \downarrow 0$ such that this is finite. Dividing by $Q_{11}^{(n)}$, both conditions (ii) and (iii) of Theorem 3.2 will be satisfied. This completes the proof.

\section{Example 3: long-range dependence in financial time series}

Let $\left(P_{n},-\infty<n<\infty\right)$ be the price of some financial asset, and let $X_{n}=\log P_{n}$. It is an established assumption that the log returns, $r_{n}=X_{n}-X_{n-1}$, are well modeled by a martingale difference process. Such a model accounts for the fact that the log returns exhibit little correlation. Nevertheless, it is also a widely observed fact that some instantaneous functions of the log returns, such as $\left|r_{n}\right|^{d}$, exhibit long memory (see, e.g. [6]).

The popular approach to modeling this behavior has been to explicitly incorporate the dependence of the absolute log returns into the statistical description of the model. The result is the various long-memory, autoregressive conditional heteroskedasticity (ARCH) process models of financial time series (see, e.g. [9]).

We want to show in this example that, given a martingale difference sequence $\left(r_{n}\right)$ that can be represented as a function of an LRD Markov chain, the outcome that $\left|r_{n}\right|^{d}$ will exhibit long-range dependence should not be considered surprising.

We want to illustrate this with a very simple example based on Mandelbrot's model for wheat prices (see [11]). We note that this simple model is for purposes of illustration only, 
and does not account for all known properties of financial time series. For instance, it has been observed in many situations that $\left(r_{n}\right)$ has a finite variance, despite having a polynomially decaying marginal distribution. The $\left(r_{n}\right)$ in this example have infinite variance. Nevertheless, the proof scheme used here to establish the long-range dependence of $\left|r_{n}\right|^{d}$ should be applicable much more generally.

Let $\left(W_{n}\right)$ be a stationary random process which models the weather. The process $\left(W_{n}\right)$ can take on three values: good, bad, and neutral, denoted by $\{g, b, n\}$. The length of a good period, $T$ (number of consecutive good days), has the same distribution as the length of a bad or a neutral period. Let $\mathrm{P}(T \geq t)=t^{-\alpha}$. The period $T$ has finite mean but infinite variance (i.e. $1<\alpha \leq 2$ ). A good or bad period is followed necessarily by a neutral period. A neutral period is followed by a good or bad period with equal probability.

Let $\hat{X}_{n}$ be the fundamental (log) price of the asset (which can be thought of as summarizing exogenous variables that affect the real price). The variation of $\hat{X}_{n}$ is such that it increases by 1 for every good day, decreases by 1 for every bad day, and stays the same for every neutral day. The market calculates the real $(\log )$ price by projecting the expected future fundamental price:

$$
X_{n}=\lim _{t \rightarrow \infty} \mathrm{E}\left[\hat{X}_{n+t} \mid \hat{X}_{-\infty}^{n}\right]
$$

By construction, $\left(r_{n}\right)$ is itself a martingale difference sequence. We will now show that, $\varrho_{n}=\left|r_{n}\right|^{d}$ is LRD with Hurst index $\frac{1}{2}(3-\alpha)$. (For $\operatorname{var}\left(\varrho_{0}\right)$ to be finite $0<d<\alpha / 2$.)

It can be verified (see also the calculations in Mandelbrot's original paper [11]) that $X_{n}$ changes as follows: jumps by $\mathrm{E}[T]$ on the first good day, jumps by $-\mathrm{E}[T]$ on the first bad day, increases by $\mathrm{E}[T \mid T \geq t]-\mathrm{E}[T \mid T \geq t-1]$ on the $t$ th good day $(t \geq 2)$, and decreases by $\mathrm{E}[T \mid T \geq t]-\mathrm{E}[T \mid T \geq t-1]$ on the $t$ th bad day. The first neutral following $t \operatorname{good}$ days decreases $X_{n}$ by E $[T \mid T \geq t]-t$. The first neutral following $t$ bad days increases $X_{n}$ by $\mathrm{E}[T \mid T \geq t]-t$.

Let $J_{n}=1$ (there is a transition at time $n$ ). Let

$$
T_{n}:=\inf _{t}\left\{t \geq 0: W_{n-t-1} \neq W_{n-t-2}\right\}
$$

be the number of days since the last transition ( 0 on the first day following).

Then $M_{n}=\left(W_{n}, J_{n}, T_{n}\right)$ is a countable state, LRD Markov chain, with Hurst index $\frac{1}{2}(3-\alpha)$. Moreover, $\varrho_{n}=\left|r_{n}\right|^{d}$ is a function of $M_{n}$ :

- $\rho(\{g, b\}, 0, t)=(\mathrm{E}[T \mid T \geq t+2]-\mathrm{E}[T \mid T \geq t+1])^{d}$,

- $\rho(\{n\}, 0, \cdot)=0$,

- $\rho(\{g, b\}, 1, \cdot)=(\mathrm{E}[T])^{d}$,

- $\rho(\{n\}, 1, t)=(\mathrm{E}[T \mid T \geq t+1]-(t+1))^{d}$.

Lemma 6.1. It holds that

$$
\mathrm{E}[T \mid T \geq t+2]-\mathrm{E}[T \mid T \geq t+1] \rightarrow \frac{\alpha}{\alpha-1} \quad \text { as } t \rightarrow \infty .
$$

Proof. We have

$$
\mathrm{P}(T \geq s \mid T \geq t)=\frac{s^{-\alpha}}{t^{-\alpha}}, \quad s \geq t,
$$


and

$$
\begin{aligned}
\mathrm{E}[T \mid T \geq t+1]-\mathrm{E}[T \mid T \geq t] & =\sum_{s=t+1}^{\infty} \mathrm{P}(T \geq s \mid T \geq t+1)-\mathrm{P}(T \geq s \mid T \geq t) \\
& =\left((t+1)^{\alpha}-t^{\alpha}\right) \sum_{s=t+1}^{\infty} s^{-\alpha} \\
& \rightarrow \frac{\alpha}{\alpha-1},
\end{aligned}
$$

since

$$
\frac{1}{\alpha-1}(t+2)^{-\alpha+1}=\int_{t+2}^{\infty} s^{-\alpha} \mathrm{d} s<\sum_{s=t+1}^{\infty} s^{-\alpha}<\int_{t+1}^{\infty} s^{-\alpha} \mathrm{d} s=\frac{1}{\alpha-1}(t+1)^{-\alpha+1}
$$

and $\left((t+1)^{\alpha}-t^{\alpha}\right) / t^{\alpha-1} \rightarrow \alpha$. This completes the proof.

Lemma 6.2. It holds that

$$
\mathrm{E}[T \mid T \geq t]-t \leq \frac{t}{\alpha-1}
$$

Proof. We have

$$
\mathrm{E}[T \mid T \geq t]-t=\sum_{s=t}^{\infty} \frac{s^{-\alpha}}{t^{-\alpha}} \leq \int_{t}^{\infty} s^{-\alpha} \mathrm{d} s=\frac{t}{\alpha-1},
$$

completing the proof.

We will utilize Theorem 3.2 with $\mathcal{A}_{1}=(\{g, b\}, 0, \cdot), \mathcal{A}_{2}=(\{n\}, 0, \cdot), \mathcal{A}_{3}=(\{g, b\}, 1, \cdot)$, $\mathcal{A}_{4}=(\{n\}, 1, \cdot), c_{1}=c_{4}=(\alpha /(\alpha-1))^{d}, c_{2}=c_{3}=0$, and $\mathcal{H}=(\cdot, \cdot, 0)$. We have

$$
\begin{aligned}
\operatorname{var}\left(\varrho_{0}\right) & \leq \mathrm{E} \varrho_{0}^{2} \\
& =\sum_{i} \pi_{i} \rho(i)^{2} \\
& =\sum_{i \notin \mathcal{A}_{4}} \pi_{i} \rho(i)^{2}+\sum_{i \in \mathcal{A}_{4}} \pi_{i} \rho(i)^{2} \\
& \leq C+\sum_{t=1}^{\infty} \frac{1}{2} \mathrm{P}(T=t)\left(\frac{t}{\alpha-1}\right)^{2 d} \\
& <\infty
\end{aligned}
$$

by Lemma 6.2. As $\rho(i)$ is bounded when $i \notin \mathcal{A}_{4}$, the contribution to the sum is a constant $C$. We also used the fact that if $i=(\{n\}, 1, t-1)$ then $\pi_{i}=\mathrm{P}\left(W_{-t}=n\right) \mathrm{P}(T=t)=\frac{1}{2} \mathrm{P}(T=t)$.

We need to first show that Theorem 3.2(i) holds:

$$
\lim _{n \rightarrow \infty} \frac{1}{Q_{11}^{(n)}} \sum_{r=1}^{n} \sum_{i \in \mathcal{A}_{k}, j \in \mathcal{A}_{l}} \pi_{i}|\rho(i)-\mu||\rho(j)-\mu|_{\mathcal{H}} p_{i j}^{(r)} \rightarrow 0 \quad \text { for all } k \neq l .
$$

By inspection, the following transitions require visiting $\mathscr{H}:(k, l)$ or $(l, k)=(1,2),(1,3)$, $(2,4),(3,4)$. The sum is 0 for these pairs. For $(k, l)$ or $(l, k)=(1,4),(2,3)$, the condition is not needed due to the note at the end of the statement of Theorem 3.2(ii). 
Theorem 3.2(ii) reads

$$
\lim _{n \rightarrow \infty} \frac{1}{Q_{11}^{(n)}} \sum_{i, j \in \mathcal{A}_{k}} \pi_{i}\left(\rho(i)-c_{k}\right)\left(\rho(j)-c_{k}\right) \sum_{r=1}^{n} \mathscr{H}_{i j}^{(r)}=0 \quad \text { for all } k .
$$

For $k=3,4,{ }_{\mathscr{H}} p_{i j}^{(r)}=0$ because these states must go to $\mathscr{H}$ in one step. For $k=1,2$, we have chosen $c_{k}$ such that $\rho(i)-c_{k} \rightarrow 0$ by Lemma 6.1. The condition holds by remark 2 .

Theorem 3.2(iii) also holds for $\mathcal{A}_{1}, \mathcal{A}_{2}$, and $\mathcal{A}_{3}$ because $\rho$ is bounded on these sets. On $\mathcal{A}_{4}$, it holds because ${ }_{\mathcal{H}} p_{i j}^{(r)}=0$, as argued earlier. We finally have the conclusion:

$$
\lim _{n \rightarrow \infty} \frac{\sum_{r=1}^{n} \operatorname{cov}\left(\left|r_{0}\right|^{d},\left|r_{n}\right|^{d}\right)}{Q_{11}^{(n)} / \pi_{1}}=\sum_{k=1}^{K} \pi_{k}^{\infty}\left(\mu-c_{k}\right)^{2}>0 .
$$

\section{A nonexample}

Consider an LRD Markov chain with $p_{12}^{(1)}=1$ and $p_{i 2}^{(1)}=0$ for $i>1$. Set $\rho(1)=1$, $\rho(2)=-1$, and $\rho(i)=0$ for $i>2$. We have for this chain $\pi_{1}=\pi_{2}$ and $\mu=0$. Since $\rho(i)=0$ for $i>2$, the conditions of Theorem 3.1 hold with $c=0$ and $\mathscr{H}=\{1\}$. However, since $\mu=c$, the conclusion about the equality of the Hurst indices does not follow. In fact, we can show $\varrho$ to be short-range dependent. From [5, Section 3] we know that

$$
\sum_{r=1}^{n} \operatorname{cov}\left(\varrho_{0}, \varrho_{r}\right)=\sum_{i, j} \rho(i) \rho(j) \pi_{i} Q_{i j}^{(n)} .
$$

The right-hand side is a finite sum, giving

$$
\sum_{r=1}^{n} \operatorname{cov}\left(\varrho_{0}, \varrho_{r}\right)=\pi_{1}\left(Q_{11}^{(n)}+Q_{22}^{(n)}\right)-\pi_{1}\left(Q_{12}^{(n)}+Q_{21}^{(n)}\right)
$$

where we have used the fact that $\pi_{1}=\pi_{2}$. Since $p_{12}^{(1)}=1$ and $p_{i 2}^{(1)}=0$ for $i>1$, we also know that $p_{12}^{(r+1)}=p_{11}^{(r)}$ and $p_{22}^{(r+1)}=p_{21}^{(r)}$. Expanding the $Q^{(n)}$ as sums, we obtain

$$
\begin{aligned}
\sum_{r=1}^{n} \operatorname{cov}\left(\varrho_{0}, \varrho_{r}\right) & =\pi_{1} \sum_{r=1}^{n}\left(p_{12}^{(r+1)}-\pi_{1}\right)-\left(p_{12}^{(r)}-\pi_{1}\right)+\pi_{1} \sum_{r=1}^{n}\left(p_{22}^{(r)}-\pi_{1}\right)-\left(p_{22}^{(r+1)}-\pi_{1}\right) \\
& =\pi_{1}\left[\left(p_{12}^{(n+1)}-\pi_{1}\right)+\left(p_{22}^{(1)}-\pi_{1}\right)-\left(p_{12}^{(1)}-\pi_{1}\right)-\left(p_{22}^{(n+1)}-\pi_{1}\right)\right],
\end{aligned}
$$

which remains bounded, demonstrating that $\left.\varrho_{n}\right)$ is a short-range-dependent process.

\section{Proofs of the theorems}

For the proofs, we will rely on several lemmas, most of which are already known.

Lemma 8.1. ([5, Corollary 1, Chapter 11].) For $p \geq 0$,

$$
\mathrm{E}_{1} T_{1}^{p}=\infty \quad \Longleftrightarrow \quad \mathrm{E}_{i} T_{i}^{p}=\infty \text { for all } i \in \mathbb{N} .
$$

Lemma 8.2. Let $\left(a_{n}\right)$ be an arbitrary sequence, and let $b_{n} \rightarrow \infty$. Let c be a finite real number. If

$$
\frac{a_{n}}{b_{n}} \rightarrow c
$$


then

$$
\frac{\sum_{r=1}^{n} a_{r}}{\sum_{r=1}^{n} b_{r}} \rightarrow c .
$$

Proof. This elementary result follows from the discrete analogue of l'Hôpital's rule, referred to as the Stolz-Cesàro theorem.

Lemma 8.3. (i) $\operatorname{cov}\left(\varrho_{0}, \varrho_{r}\right)=\sum_{i, j} \pi_{i} p_{i j}^{(r)}(\rho(i)-\mu)(\rho(j)-\mu)$.

(ii) $\sum_{r=1}^{n} \operatorname{cov}\left(\varrho_{0}, \varrho_{r}\right)=\sum_{i, j} \rho(i) \rho(j) \pi_{i} Q_{i j}^{(n)}$.

(iii) $\operatorname{var}\left(\varrho_{0}+\cdots+\varrho_{n}\right)-(n+1) \operatorname{var}\left(\varrho_{0}\right)=2 \sum_{i, j} \rho(i) \rho(j) \pi_{i} R_{i j}^{(n)}$.

Proof. Part (i) is a simple expansion. Part (ii) is derived from (i), and (iii) can be found in [4, Section 3].

Lemma 8.4. ([5, Equation (1), Theorem 9.1].) It holds that

$$
p_{i j}^{(r)}={ }_{1} p_{i j}^{(r)}+\sum_{m=1}^{r-1}{ }_{1} p_{i 1}^{(m)} p_{1 j}^{(r-m)}, \quad r \geq 1 .
$$

Lemma 8.5. ([4, Equation (12)].) It holds that

$$
\begin{aligned}
Q_{11}^{(n)} & \sim\left(\pi_{1}\right)^{2} \sum_{u=1}^{\infty} \min (u, n) \sum_{s=u+1}^{\infty} f_{11}^{(s)} \\
& =\left(\pi_{1}\right)^{2} \sum_{u=1}^{\infty} \sum_{r=1}^{\min (u, n)} \sum_{s=u+1}^{\infty} f_{11}^{(s)} \\
& =\left(\pi_{1}\right)^{2} \sum_{r=1}^{n} \sum_{u=r}^{\infty} \sum_{s=u+1}^{\infty} f_{11}^{(s)} .
\end{aligned}
$$

Lemma 8.6. It holds that

$$
\lim _{n \rightarrow \infty} \frac{1}{Q_{11}^{(n)} / \pi_{1}} \sum_{i, j} \pi_{i} \sum_{r=1}^{n}{ }_{1} p_{i j}^{(r)}=1 .
$$

Proof. We have

$$
\begin{aligned}
\sum_{i, j} \pi_{i} \sum_{r=1}^{n}{ }_{1} p_{i j}^{(r)} & =\sum_{r=1}^{n} \sum_{i, j} \pi_{i} p_{i j}^{(r)} \\
& =\sum_{r=1}^{n} \sum_{i} \pi_{i} \sum_{u=r}^{\infty} f_{i 1}^{(r)} \\
& =\sum_{r=1}^{n} \sum_{u=r}^{\infty} \frac{1}{m_{11}} \sum_{s=u}^{\infty} f_{11}^{(s)} \\
& =\frac{1}{m_{11}} \sum_{r=1}^{n} \sum_{u=r}^{\infty} f_{11}^{u}+\sum_{r=1}^{n} \sum_{u=r}^{\infty} \frac{1}{m_{11}} \sum_{s=u+1}^{\infty} f_{11}^{(s)}
\end{aligned}
$$




$$
\begin{aligned}
& =\frac{1}{m_{11}} \sum_{r=1}^{n} \mathrm{P}_{1}\left(T_{1} \geq r\right)+\sum_{r=1}^{n} \sum_{u=r}^{\infty} \frac{1}{m_{11}} \sum_{s=u+1}^{\infty} f_{11}^{(s)} \\
& \sim \frac{1}{\pi_{1}^{2} m_{11}} Q_{11}^{(n)} \\
& =\frac{Q_{11}^{(n)}}{\pi_{1}},
\end{aligned}
$$

since $\sum_{r=1}^{n} \mathrm{P}_{1}\left(T_{1} \geq r\right) \leq m_{11}$ and by Lemma 8.5. In the second equality we have used the fact that $\sum_{j{ }_{1}} p_{i j}^{(r)}=\sum_{r}^{\infty} f_{i 1}^{(r)}$, which is an equivalent way of expressing the probability of going from $i$ to any other state without going to 1 in $r$ steps. This expression also appears in Chapter 9 of [5] (see the proof of Theorem 6 therein). In the third equality we have used the fact that $\mathrm{P}_{\pi}\left(T_{1}=r\right)=\mathrm{P}_{1}\left(T_{1} \geq r\right) / m_{11}$, where $T_{1}$ is the first return time to 1 at stationarity. This completes the proof.

Lemma 8.7. Let $M>0$ be a finite number. Then

$$
\lim _{n \rightarrow \infty} \frac{1}{Q_{11}^{(n)} / \pi_{1}} \sum_{\{i<M\} \cup\{j<M\}} \pi_{i} \sum_{r=1}^{n}{ }_{1} p_{i j}^{(r)}=0 .
$$

Proof. Pick $m$ such that ${ }_{1} p_{1 i}^{(m)}>0$. Then

$$
{ }_{1} p_{1 i}^{(m)}{ }_{1} p_{i j}^{*} \leq{ }_{1} p_{1 j}^{*}=\frac{\pi_{j}}{\pi_{1}} .
$$

Thus, there exists a finite constant $C_{M}$ such that ${ }_{1} p_{i j}^{*}<C_{M} \pi_{j}$ for all $i<M$. Therefore,

$$
\sum_{i<M, j} \pi_{i} \sum_{r=1}^{n}{ }_{1} p_{i j}^{(r)} \leq C_{M} \sum_{i<M, j} \pi_{i} \pi_{j} \leq C_{M} .
$$

Similarly, there exists a finite constant $D_{M}$ such that ${ }_{1} p_{i j}^{*} \leq 1+{ }_{1} p_{j j}^{*} \leq D_{M}$ for all $j<M$. Hence,

$$
\sum_{j<M, i} \pi_{i} \sum_{r=1}^{n}{ }_{1} p_{i j}^{(r)} \leq D_{M} \sum_{j<M, i} \pi_{i} \leq M D_{M} .
$$

Using (2.1) completes the proof.

Lemma 8.8. ([4, p. 1051].) It holds that

$$
\left|\frac{Q_{1 j}^{(n)} / \pi_{j}}{Q_{11}^{(n)} / \pi_{1}}\right| \leq 1
$$

Lemma 8.9. It holds that

$$
\begin{aligned}
& \left.\left|\sum_{r=1}^{n} \sum_{i, j} \pi_{i}\right| \rho(i) \rho(j)\right|_{1} p_{i j}^{(r)}-\sum_{r=1}^{n} \sum_{i, j} \pi_{i}|\rho(i) \rho(j)|_{\mathscr{H}} p_{i j}^{(r)} \mid \\
& \quad \leq(|\mathscr{H}|+1) C_{\mathcal{H}} \sum_{i, j} \pi_{i} \pi_{j}|\rho(i) \rho(j)|,
\end{aligned}
$$

where $\mathcal{H}$ is any nonempty set with a finite number of states and $C_{\mathcal{H}}$ is a constant that depends only on $\mathcal{H}$. 
Proof. Let $\mathscr{H}^{\prime}=\mathscr{H} \cup\{k\}, k \notin \mathscr{H}$. We will argue by induction. We write

$$
\begin{aligned}
\sum_{r=1}^{n}{ }_{\mathcal{H}} & p_{i j}^{(r)}-{ }_{\mathcal{H}^{\prime}} p_{i j}^{(r)} \\
& =\sum_{r=1}^{n} \mathrm{P}\left(M_{r}=j ; M_{l} \notin \mathscr{H}, 1 \leq l<r ; M_{l}=k \text { for some } 1 \leq l<r \mid M_{0}=i\right) \\
& =\sum_{r=1}^{n} \sum_{m=1}^{r-1} \mathscr{H}^{\prime} p_{i k}^{(m)} \mathscr{H}_{k j}^{(r-m)} \\
& =\sum_{m=1}^{n-1} \operatorname{H}^{\prime} p_{i k}^{(m)} \sum_{r=m+1}^{n}{ }_{\mathcal{H}}^{(r-m)} p_{k j}^{(r-m)} \\
\leq & \underbrace{\left(\sum_{m=1}^{\infty} \mathscr{H}^{\prime} p_{i k}^{(m)}\right)}_{C_{1}} \underbrace{\left(\sum_{\mathscr{H}}^{\infty} p_{k j}^{(r)}\right)}_{\mathscr{H} p_{k j}^{*}} .
\end{aligned}
$$

The constant $C_{1}$ is bounded above by 1 since

$$
\sum_{m=1}^{\infty} \mathscr{H}^{\prime} p_{i k}^{(m)} \leq \sum_{m=1}^{\infty}{ }_{k} p_{i k}^{(m)}=1
$$

Let $h \in \mathscr{H}$. Choose $m$ such that ${ }_{h} p_{h k}^{(m)}>0$. Then

$$
{ }_{h} p_{h k}^{(m)}{ }_{H} p_{k j}^{*} \leq{ }_{h} p_{h j}^{*}=\frac{\pi_{j}}{\pi_{h}} .
$$

Thus, ${ }_{\mathscr{H}} p_{k j}^{*} \leq \pi_{j} /\left({ }_{h} p_{h k}^{(m)} \pi_{h}\right)=C_{\mathscr{H}^{\prime}}$. Therefore,

$$
\sum_{r=1}^{n} \sum_{i, j} \pi_{i}|\rho(i) \rho(j)|_{\mathscr{H}} p_{i j}^{(r)}-\sum_{r=1}^{n} \sum_{i, j} \pi_{i}|\rho(i) \rho(j)|_{\mathscr{H}^{\prime}} p_{i j}^{(r)} \leq C_{\mathscr{H}^{\prime}} \sum_{i, j} \pi_{i} \pi_{j}|\rho(i) \rho(j)| .
$$

Therefore, adding or subtracting a state from the set $\mathscr{H}$ (as long as the resulting set is nonempty) only affects the sum in question by a bounded amount. As a result, replacing $\mathscr{H}$ by $\{1\}$ can change the sum by at most $(1+|\mathscr{H}|) C_{\mathscr{H}} \sum_{i, j} \pi_{i} \pi_{j}|\rho(i) \rho(j)|$. (Add state 1 if it is not already in set $\mathcal{H}$. Then subtract all other states until only state 1 is left.) This completes the proof.

\subsection{Proof of Theorem 3.1}

By (2.1) and Lemma 8.9, conditions (i) and (ii) of Theorem 3.1 are respectively equivalent to

$$
\lim _{n \rightarrow \infty} \frac{1}{Q_{11}^{(n)} / \pi_{1}} \sum_{r=1}^{n} \sum_{i, j} \pi_{i}(\rho(i)-c)(\rho(j)-c)_{1} p_{i j}^{(r)}=0
$$

for some constant $c$ and

$$
\lim _{L \rightarrow \infty} \limsup _{n \rightarrow \infty} \frac{1}{Q_{11}^{(n)} / \pi_{1}} \sum_{r=1}^{n} \sum_{i, j} \pi_{i}|\rho(i) \rho(j)| 1(|\rho(i)|,|\rho(j)|>L)_{1} p_{i j}^{(r)}=0 .
$$


Define

$$
\bar{\rho}^{M}(i)= \begin{cases}\rho(i), & i \leq M \\ c, & i>M,\end{cases}
$$

$\bar{\mu}^{M}=\mathrm{E}\left[\bar{\varrho}_{n}^{M}\right], \underline{\rho}^{M}(i)=\rho(i)-\bar{\rho}^{M}(i)$, and $\underline{\mu}^{M}=\mathrm{E}\left[\underline{\varrho}_{n}^{M}\right]$. We adopt the shorthand notation

$$
\phi_{n}=\frac{\varrho_{0}+\cdots+\varrho_{n}-(n+1) \mu}{\sqrt{2 R_{11}^{(n)} / \pi_{1}}}, \quad \bar{\phi}_{n}^{M}=\frac{\bar{\varrho}_{0}^{M}+\cdots+\bar{\varrho}_{n}^{M}-(n+1) \bar{\mu}^{M}}{\sqrt{2 R_{11}^{(n)} / \pi_{1}}},
$$

and

$$
\underline{\phi}_{n}^{M}=\phi_{n}-\bar{\phi}_{n}^{M}
$$

We will be referring to the reverse triangle inequality for random variables:

$$
\left|\sqrt{\operatorname{var}\left(\phi_{n}\right)}-\sqrt{\operatorname{var}\left(\bar{\phi}_{n}^{M}\right)}\right| \leq \sqrt{\operatorname{var}\left(\underline{\phi}_{n}^{M}\right)} .
$$

Using Lemma 8.4, write Lemma 8.3(i) as

$$
\begin{aligned}
\sum_{r=1}^{n} \operatorname{cov}\left(\varrho_{0}, \varrho_{r}\right)= & \sum_{i, j} \pi_{i}(\rho(i)-\mu)(\rho(j)-\mu) \sum_{r=1}^{n}{ }_{1} p_{i j}^{(r)} \\
& +\sum_{i, j} \pi_{i} \sum_{r=1}^{n} \sum_{m=1}^{r-1}{ }_{1} p_{i 1}^{(m)} p_{1 j}^{(r-m)}(\rho(i)-\mu)(\rho(j)-\mu) .
\end{aligned}
$$

The second term can be rewritten as

$$
\begin{aligned}
\sum_{i, j} \pi_{i} & \sum_{r=1}^{n} \sum_{m=1}^{r-1}{ }_{1} p_{i 1}^{(m)} p_{1 j}^{(r-m)}(\rho(i)-\mu)(\rho(j)-\mu) \\
= & \sum_{i, j} \pi_{i} \sum_{m=1}^{n-1}{ }_{1} p_{i 1}^{(m)} \sum_{r=m+1}^{n} p_{1 j}^{(r-m)}(\rho(i)-\mu)(\rho(j)-\mu) \\
= & \sum_{m=1}^{n-1}\left(\sum_{r=m+1} \sum_{i, j}{ }_{1} p_{i 1}^{(m)} \pi_{i}\left(p_{1 j}^{(r-m)}-\pi_{j}\right)(\rho(i)-\mu)(\rho(j)-\mu)\right. \\
& \quad+\underbrace{\sum_{r=m+1}^{n} \sum_{i, j} p_{i 1}^{(m)} \pi_{i} \pi_{j}(\rho(i)-\mu)(\rho(j)-\mu)}) \\
= & \sum_{m=1}^{n-1} \sum_{i, j} \pi_{i} p_{i 1}^{(m)} Q_{1 j}^{(n-m)}(\rho(i)-\mu)(\rho(j)-\mu) .
\end{aligned}
$$

Dividing by $Q_{11}^{(n)} / \pi_{1}$ we obtain

$$
\sum_{m=1}^{n-1} \sum_{i, j} \pi_{i 1} p_{i 1}^{(m)} \pi_{j} \frac{Q_{1 j}^{(n-m)} / \pi_{j}}{Q_{11}^{(n)} / \pi_{1}}(\rho(i)-\mu)(\rho(j)-\mu) .
$$


By Lemma 8.8 we have

$$
\sum_{j} \pi_{j}\left|\frac{Q_{1 j}^{(n-m)} / \pi_{j}}{Q_{11}^{(n)} / \pi_{1}}\right||(\rho(j)-\mu)|<\infty .
$$

We also know that $\sum_{i} \pi_{i} \sum_{m=11}^{n-1} p_{i 1}^{(m)}(\rho(i)-\mu) \rightarrow 0$. Therefore,

$$
\lim _{n \rightarrow \infty} \sum_{m=1}^{n-1} \sum_{i, j} \pi_{i 1} p_{i 1}^{(m)} \pi_{j} \frac{Q_{1 j}^{(n-m)} / \pi_{j}}{Q_{11}^{(n)} / \pi_{1}}(\rho(i)-\mu)(\rho(j)-\mu)=0,
$$

by the dominated convergence theorem. The above result has the interpretation that the sum of the covariances between $\varrho_{0}$ and $\varrho_{n}$ on the event that the chain visits state 1 at least once before time $n$ is negligible compared to $Q_{11}^{(n)}$.

We want to use these results to conclude that $\operatorname{var}\left(\underline{\phi}_{n}^{M}\right) \rightarrow 0$. For this, we write (8.3) for $\underline{\rho}^{M}$, $c=0$. The first term in (8.3) reads, after a little manipulation,

$$
\sum_{i, j} \pi_{i}\left[\underline{\rho}^{M}(i) \underline{\rho}^{M}(j)-\underline{\mu}^{M}\left(\underline{\rho}^{M}(i)+\underline{\rho}^{M}(j)\right)+\left(\underline{\mu}^{M}\right)^{2}\right] \sum_{r=1}^{n}{ }_{1} p_{i j}^{(r)} .
$$

Now assume that $\rho$ is bounded. Upon dividing by $Q_{11}^{(n)} / \pi_{1}$, the second and third terms are $O\left(\underline{\mu}^{M}\right)$ as $\underline{\mu}^{M} \rightarrow 0$ by Lemma 8.6. Since $\underline{\mu}^{M} \rightarrow 0$ with $M$, these terms go to 0 as $M \rightarrow \infty$ uniformly in $n$.

For the first term in (8.4), write (8.1) as follows for comparison:

$$
\begin{aligned}
& \lim _{n \rightarrow \infty} \frac{1}{Q_{11}^{(n)} / \pi_{1}}\left(\sum_{r=1}^{n} \sum_{i \leq M, j \leq M} \pi_{i}(\rho(i)-c)(\rho(j)-c){ }_{1} p_{i j}^{(r)}\right. \\
&+\sum_{r=1}^{n} \sum_{i \leq M, j>M} \pi_{i}(\rho(i)-c)(\rho(j)-c)_{1} p_{i j}^{(r)} \\
&+\sum_{r=1}^{n} \sum_{i>M, j \leq M} \pi_{i}(\rho(i)-c)(\rho(j)-c){ }_{1} p_{i j}^{(r)} \\
&\left.+\sum_{r=1}^{n} \sum_{i>M, j>M} \pi_{i}(\rho(i)-c)(\rho(j)-c)_{1} p_{i j}^{(r)}\right) \\
&= 0 .
\end{aligned}
$$

The first three sums have limit 0 owing to the fact that $\rho$ is assumed to be bounded and using Lemma 8.7. The last sum is identical to the first term in (8.4). Therefore, dividing (8.3) by $Q_{11}^{(n)} / \pi_{1}$ and applying Lemma 8.2, together with Lemma 8.3(ii) and (iii), we conclude that $\lim _{M \rightarrow \infty} \lim _{n \rightarrow \infty} \operatorname{var}\left(\underline{\phi}_{n}^{M}\right)=0$, and, by (8.2), also that $\lim _{M \rightarrow \infty} \lim _{n \rightarrow \infty} \operatorname{var}\left(\bar{\phi}_{n}^{M}\right)=$ $\lim _{n \rightarrow \infty} \operatorname{var}\left(\phi_{n}\right)$.

To calculate $\operatorname{var}\left(\bar{\phi}_{n}^{M}\right)$, rewrite (8.3) for $\bar{\rho}^{M}$ :

$$
\sum_{i, j} \pi_{i}\left[\left(\bar{\rho}^{M}(i)-c\right)\left(\bar{\rho}^{M}(j)-c\right)-\left(\bar{\mu}^{M}-c\right)\left(\bar{\rho}^{M}(i)+\bar{\rho}^{M}(j)-2 c\right)+\left(\bar{\mu}^{M}-c\right)^{2}\right] \sum_{r=1}^{n}{ }_{1} p_{i j}^{(r)} .
$$


The first two sums will go to 0 when dividing by $Q_{11}^{(n)} / \pi_{1}$, by the boundedness of $\rho$ and Lemma 8.7 because of truncation. The last term will read

$$
\left(\bar{\mu}^{M}-c\right)^{2} \frac{1}{Q_{11}^{(n)} / \pi_{1}} \sum_{i, j} \pi_{i} \sum_{r=1}^{n}{ }_{1} p_{i j}^{(r)} \rightarrow\left(\bar{\mu}^{M}-c\right)^{2} \quad \text { as } n \rightarrow \infty
$$

by Lemma 8.6. By Lemma 8.3(ii) and (iii), and Lemma 8.2, this concludes the proof when $\left(\varrho_{n}\right)$ is bounded.

When $\left(\varrho_{n}\right)$ is not bounded, we truncate by value, i.e. $\tilde{\rho}^{L}(i)=\rho(i) 1(\rho(i) \leq L), \tilde{\mu}^{L}=\mathrm{E}\left[\tilde{\varrho}_{n}^{L}\right]$, ${\stackrel{\rho}{\rho^{L}}}^{L}(i)=\rho(i)-\tilde{\rho}^{L}(i)$, and ${\underset{\sim}{\mu}}^{L}=\mathrm{E}\left[\varrho_{n}^{L}\right]$. Also, define

$$
\tilde{\phi}_{n}^{L}=\frac{\tilde{\varrho}_{0}^{L}+\cdots+\tilde{\varrho}_{n}^{L}-(n+1) \tilde{\mu}^{L}}{\sqrt{2 R_{11}^{(n)} / \pi_{1}}}, \quad \phi_{n}^{L}=\phi_{n}-\tilde{\phi}_{n}^{L} .
$$

We can express $\sum_{r=1}^{n} \operatorname{cov}\left(\varrho_{0}^{L}, \varrho_{r}^{L}\right)$ as in (8.3), and argue as there that the second term has limit 0 as $n \rightarrow \infty$ when divided by $Q_{11}^{(n)} / \pi_{1}$. The first term also has limit 0 due to the assumed condition (ii). We appeal again to Lemma 8.3(ii) and (iii), and Lemma 8.2, to argue that $\lim _{L \rightarrow \infty} \lim _{n \rightarrow \infty} \operatorname{var}\left(\phi_{n}^{L}\right)=0$. By (8.2), we also obtain $\lim _{L \rightarrow \infty} \lim _{n \rightarrow \infty} \operatorname{var}\left(\tilde{\phi}_{n}^{L}\right)=$ $\lim _{n \rightarrow \infty} \operatorname{var}\left(\phi_{n}\right)$. We conclude that

$$
\lim _{n \rightarrow \infty} \frac{\operatorname{var}\left(\sum_{r=1}^{n} \varrho_{i}\right)}{R_{11}^{(n)} / \pi_{1}}=\lim _{L \rightarrow \infty} \lim _{n \rightarrow \infty} \operatorname{var}\left(\tilde{\phi}_{n}^{L}\right)=\lim _{L \rightarrow \infty}(\tilde{\mu}-c)^{2}=(\mu-c)^{2} .
$$

The claim about the Hurst indices can be argued as follows. Consider the expression in Lemma 8.3(ii) for $\varrho_{n}=1\left(M_{n}=1\right)$. Dividing by $Q_{11}^{(n)} / \pi_{1}$, we see that the right-hand side has limit $\pi_{1}^{2}>0$. From the above argument, it follows that $\left(\sum_{r=1}^{n} \operatorname{cov}\left(1\left(M_{0}=1\right)\right.\right.$, $\left.\left.1\left(M_{r}=1\right)\right)\right) /\left(\sum_{r=1}^{n} \operatorname{cov}\left(\varrho_{0}, \varrho_{r}\right)\right)$ has a finite, nonzero limit if $\mu \neq c$. It is easily seen from the definition of $H$ that $\rho$ has the same Hurst index as the indicator function $1\left(M_{n}=1\right)$. This completes the proof.

\subsection{Proof of Theorem 3.2}

By (2.1) and Lemma 8.9, conditions (i)-(iii) are respectively equivalent to

$$
\lim _{n \rightarrow \infty} \frac{1}{Q_{11}^{(n)} / \pi_{1}} \sum_{r=1}^{n} \sum_{i \in \mathcal{A}_{k}, j \in \mathcal{A}_{l}} \pi_{i}|\rho(i)-\mu||\rho(j)-\mu|_{1} p_{i j}^{(r)}=0
$$

for all $k \neq l$,

$$
\lim _{n \rightarrow \infty} \frac{1}{Q_{11}^{(n)} / \pi_{1}} \sum_{r=1}^{n} \sum_{i, j \in \mathcal{A}_{k}} \pi_{i}\left(\rho(i)-c_{k}\right)\left(\rho(j)-c_{k}\right)_{1} p_{i j}^{(r)}=0
$$

for all $k$, and

$$
\lim _{L \rightarrow \infty} \limsup _{n \rightarrow \infty} \frac{1}{Q_{11}^{(n)} / \pi_{1}} \sum_{r=1}^{n} \sum_{i, j \in \mathcal{A}_{k}} \pi_{i}|\rho(i) \rho(j)| 1(|\rho(i)|,|\rho(j)|>L)_{1} p_{i j}^{(r)}=0
$$

for all $k$. We truncate as follows:

$$
\bar{\rho}^{M}(i)= \begin{cases}\rho(i), & i<M, \\ c_{k}, & i \geq M, i \in \mathcal{A}_{k} .\end{cases}
$$

Define $\underline{\rho}^{M}, \bar{\mu}^{M}, \underline{\mu}^{M}, \bar{\phi}^{M}$, and $\underline{\phi}^{M}$ as before. 
The first sum in (8.3) can be decomposed as

$$
\begin{aligned}
\sum_{i, j} \pi_{i}(\rho(i)-\mu)(\rho(j)-\mu) \sum_{r=1}^{n}{ }_{1} p_{i j}^{(r)} & \\
= & \sum_{k=1}^{K} \sum_{i, j \in \mathcal{A}_{k}} \pi_{i}(\rho(i)-\mu)(\rho(j)-\mu) \sum_{r=1}^{n}{ }_{1} p_{i j}^{(r)} \\
& +\sum_{k, l \in\{1, \ldots, K\}, k \neq l} \sum_{i \in \mathcal{A}_{k}, j \in \mathcal{A}_{l}} \pi_{i}(\rho(i)-\mu)(\rho(j)-\mu) \sum_{r=1}^{n}{ }_{1} p_{i j}^{(r)} .
\end{aligned}
$$

The first condition ensures that the cross terms on the right-hand side are insignificant. Therefore, we can work with each subset separately.

We will argue as in the proof of Theorem 3.1 to show that $\operatorname{var}\left(\phi_{n}^{M}\right) \rightarrow 0$. The analogue of (8.4) for each of the remaining sums reads

$$
\sum_{i, j \in \mathcal{A}_{k}} \pi_{i}\left[\underline{\rho}^{M}(i) \underline{\rho}^{M}(j)-\underline{\mu}^{M}\left(\underline{\rho}^{M}(i)+\underline{\rho}^{M}(j)\right)+\left(\underline{\mu}^{M}\right)^{2}\right] \sum_{r=1}^{n}{ }_{1} p_{i j}^{(r)} .
$$

Assume that $\rho$ is bounded. Upon dividing by $Q_{11}^{(n)} / \pi_{1}$, the second and third terms are $O\left(\mu^{M}\right)$ as $\underline{\mu}^{M} \rightarrow 0$ by Lemma 8.6. Since $\underline{\mu}^{M} \rightarrow 0$ as $M \rightarrow \infty$, these terms tend to 0 as $M \rightarrow \infty$ uniformly in $n$.

For the first term, we argue exactly as in the proof of Theorem 3.1 that condition (i), together with Lemma 8.7, implies that this term, when divided by $Q_{11}^{(n)} / \pi_{1}$, goes to 0 as $n \rightarrow \infty$. Applying Lemma 8.2, together with Lemma 8.3(ii) and (iii), we conclude that

$$
\lim _{M \rightarrow \infty} \lim _{n \rightarrow \infty} \operatorname{var}\left(\underline{\phi}_{n}^{M}\right)=0
$$

and, by (8.2), also that $\lim _{M \rightarrow \infty} \lim _{n \rightarrow \infty} \operatorname{var}\left(\bar{\phi}_{n}^{M}\right)=\lim _{n \rightarrow \infty} \operatorname{var}\left(\phi_{n}\right)$.

To calculate $\operatorname{var}\left(\bar{\phi}_{n}^{M}\right)$, rewrite (8.3) for $\bar{\rho}^{M}$. We again omit the cross sums:

$$
\sum_{i, j \in \mathcal{A}_{k}} \pi_{i}\left[\left(\bar{\rho}^{M}(i)-c_{k}\right)\left(\bar{\rho}^{M}(j)-c_{k}\right)-\left(\bar{\mu}^{M}-c_{k}\right)\left(\bar{\rho}^{M}(i)+\bar{\rho}^{M}(j)-2 c_{k}\right)+\left(\bar{\mu}^{M}-c_{k}\right)^{2}\right] \sum_{r=1}^{n}{ }_{1} p_{i j}^{(r)} .
$$

The first two sums will go to 0 due to truncation, the boundedness of $\rho$, and by Lemma 8.7, when dividing by $Q_{11}^{(n)} / \pi_{1}$. The last term will read

$$
\left(\bar{\mu}^{M}-c_{k}\right)^{2} \frac{1}{Q_{11} / \pi_{1}} \sum_{i, j \in \mathcal{A}_{k}} \pi_{i} \sum_{r=1}^{n}{ }_{1} p_{i j}^{(r)} \rightarrow \pi_{\mathcal{A}_{k}}^{\infty}\left(\bar{\mu}^{M}-c_{k}\right)^{2}
$$

by Lemma 8.6 and the definition of $\pi_{\mathcal{A}_{k}}^{\infty}$. This concludes the proof when $\left(\varrho_{n}\right)$ is bounded.

When $\left(\varrho_{n}\right)$ is not bounded, we truncate by value, i.e. $\tilde{\rho}^{L}(i)=\rho(i) 1(\rho(i) \leq L), \tilde{\mu}^{L}=\mathrm{E}\left[\tilde{\varrho}_{n}^{L}\right]$, ${\stackrel{\rho}{\rho^{L}}}^{L}(i)=\rho(i)-\tilde{\rho}^{L}(i)$, and ${\underset{\sim}{\mu}}^{L}=\mathrm{E}\left[\varrho_{n}^{L}\right]$. Also, define

$$
\tilde{\phi}_{n}^{L}=\frac{\tilde{\varrho}_{0}^{L}+\cdots+\tilde{\varrho}_{n}^{L}-(n+1) \tilde{\mu}^{L}}{\sqrt{2 R_{11}^{(n)} / \pi_{1}}}, \quad \phi_{n}^{L}=\phi_{n}-\tilde{\phi}_{n}^{L} .
$$


We also partition $\varrho_{n}^{L}$ as $\sum_{k=1}^{K} \varrho_{n}^{L} 1\left(\varrho_{n}^{L} \in \mathcal{A}_{k}\right)$. Define

$$
{ }_{k} \phi_{n}^{L}=\frac{\varrho_{0}^{L} 1\left(\varrho_{0}^{L} \in \mathcal{A}_{k}\right)+\cdots+\varrho_{n}^{L} 1\left(\varrho_{n}^{L} \in \mathcal{A}_{k}\right)-(n+1) \mathrm{E}\left[\varrho_{0}^{L} 1\left(\varrho_{0}^{L} \in \mathcal{A}_{k}\right)\right]}{\sqrt{2 R_{11}^{(n)} / \pi_{1}}} .
$$

We can express $\sum_{r=1}^{n} \operatorname{cov}\left(\varrho_{0}^{L} 1\left(\varrho_{0}^{L} \in \mathcal{A}_{k}\right), \underline{\varrho}_{r}^{L} 1\left(\varrho_{r}^{L} \in \mathcal{A}_{k}\right)\right)$ by writing (8.3) for $\rho^{L}(i) 1(\underbrace{L}_{\sim}(i) \in$ $\mathcal{A}_{k}$ ), and argue as there that the second term has limit 0 as $n \rightarrow \infty$ when divided by $Q_{11}^{(n)} / \pi_{1}$. The first term also has limit 0 due to the assumed condition (iii). We appeal again to Lemma 8.3(ii) and (iii), and Lemma 8.2 to $\operatorname{argue}$ that $\lim _{L \rightarrow \infty} \lim _{n \rightarrow \infty} \operatorname{var}\left({ }_{k} \phi_{n}^{L}\right)=0$. Applying (8.2), we conclude that

$$
\lim _{L \rightarrow \infty} \lim _{n \rightarrow \infty} \operatorname{var}\left(\phi_{n}^{L}\right)=\lim _{L \rightarrow \infty} \lim _{n \rightarrow \infty} \operatorname{var}\left(\sum_{k=1}^{K}{ }_{k} \phi_{n}^{L}\right)=0 .
$$

One more application of (8.2) gives $\lim _{L \rightarrow \infty} \lim _{n \rightarrow \infty} \operatorname{var}\left(\tilde{\phi}_{n}^{L}\right)=\lim _{n \rightarrow \infty} \operatorname{var}\left(\phi_{n}\right)$. We conclude that

$$
\begin{aligned}
\lim _{n \rightarrow \infty} \frac{\operatorname{var}\left(\sum_{r=1}^{n} \varrho_{i}\right)}{R_{11}^{(n)} / \pi_{1}} & =\lim _{M \rightarrow \infty} \lim _{n \rightarrow \infty} \operatorname{var}\left(\bar{\phi}_{n}^{M}\right) \\
& =\lim _{M \rightarrow \infty} \sum_{k=1}^{K} \pi_{\mathcal{A}_{k}}^{\infty}\left(\bar{\mu}^{M}-c_{k}\right)^{2} \\
& =\sum_{k=1}^{K} \pi_{\mathcal{A}_{k}}^{\infty}\left(\mu-c_{k}\right)^{2},
\end{aligned}
$$

where the second equality follows from the bounded version of the theorem proved above.

To prove the note given at the end of the theorem statement, consider $\mathcal{A}_{k} \cup \mathcal{A}_{l}$ as one subset. We can safely ignore the cross terms in (8.5), without needing to use condition (i) for the pair $\mathcal{A}_{k}, \mathcal{A}_{l}$. We do not use condition (i) in the remaining part of the proof.

All that remains is to note that

$$
\left(\bar{\mu}^{M}-c_{k}\right)^{2} \frac{1}{Q_{11} / \pi_{1}} \sum_{i, j \in \mathcal{A}_{k} \cup \mathcal{A}_{l}} \pi_{i} \sum_{r=1}^{n}{ }_{1} p_{i j}^{(r)} \rightarrow \pi_{\mathcal{A}_{k} \cup \mathcal{A}_{l}}^{\infty}\left(\bar{\mu}^{M}-c_{k}\right)^{2},
$$

where $\pi_{\mathcal{A}_{k} \cup \mathcal{A}_{l}}^{\infty}=\pi_{\mathcal{A}_{k}}^{\infty}+\pi_{\mathcal{A}_{l}}^{\infty}$.

\section{Conclusion}

We have provided conditions under which the growth rate of the variance of a function of a Markov chain is identical to that of the chain itself. Although our results simplify certain proofs greatly, there is still considerable art in using them. One needs to first construct a suitable Markov chain for the problem. One also needs to choose the parameters in the theorems carefully. Although the answer will ultimately be the same, picking state 1, partitioning $\left\{\mathcal{A}_{k}\right\}$ and $\left\{c_{k}\right\}$ appropriately can greatly reduce the amount of calculation required.

We do not have an answer for the case where $\rho(i)$ grows without bound as $i \rightarrow \infty$. In this case there might possibly exist examples in which the $\left(\varrho_{n}\right)$ have a higher Hurst index than the chain. The proof of Theorem 3.1 can provide insights for solving such questions. 
The usefulness of the theorems was demonstrated by various examples in three diverse fields. Many more can be contemplated in other areas, such as queue analysis in stochastic networks and agent-based models in finance.

\section{Acknowledgements}

The research of the authors was supported by the ARO MURI grant W911NF-08-1-0233, 'Tools for the Analysis and Design of Complex Multi-Scale Networks', by the NSF grant CNS-0910702, and the NSF Science and Technology Center grant CCF-0939370, 'Science of Information'.

\section{References}

[1] Barron, A. (1985). Logically smooth density estimation. Doctoral Thesis, Department of Electrical Engineering, Stanford University.

[2] Beran, J., Sherman, R., Taqqu, M. S. And Willinger, W. (1995). Long-range dependence in variable-bit-rate video traffic. IEEE Trans. Commun. 43, 1566-1579.

[3] Bingham, N. H., Goldie, C. M. And Teugels, J. L. (1989). Regular Variation. Cambridge University Press.

[4] Carpio, K. J. E. AND Daley, D. J. (2007). Long-range dependence of Markov chains in discrete time on countable state space. J. Appl. Prob. 44, 1047-1055.

[5] Chung, K. L. (1967). Markov Chains with Stationary Transition Probabilities. Springer, New York.

[6] Cont, R. (2001). Empirical properties of asset returns: stylized facts and statistical issues. Quant. Finance 1, 223-236.

[7] FitzeK, F. H. P. AND Reisslein, M. (2001). MPEG-4 and H. 263 video traces for network performance. IEEE Network 15, 40-54.

[8] Garrett, M. W. and Willinger, W. (1994). Analysis, modeling and generation of self-similar VBR video traffic. In Proc. ACM SIGCOMM '94 (London, UK), ACM, pp. 269-280.

[9] Giraitis, L., Robinson, P. M. And Surgailis, D. (2000). A model for long memory conditional heteroscedasticity. Ann. Appl. Prob. 10, 1002-1024.

[10] Kontoyiannis, I. (1997). Second-order noiseless source coding theorems. IEEE Trans. Inf. Theory 43, 13391341.

[11] Mandelbrot, B. (1966). Forecasts of future prices, unbiased markets, and "martingale" models. J. Business 39, 242-255.

[12] Minalis, G. et al. (2009). Scheduling policies for single-hop networks with heavy-tailed traffic. In Proc. 47th Annual Allerton Conf., pp. 112-120.

[13] OĞUZ, B. ANd ANANTHARAM, V. (2010). Compressing a long range dependent renewal process. In IEEE Internat. Symp. on Information Theory Proc., pp. 1443-1447.

[14] Rose, O. (1995). Statistical properties of MPEG video traffic and their impact on traffic modeling in ATM systems. In Proc. 20th Annual IEEE Conf. on Local Computer Networks, IEEE Computer Society Press, Washington, DC, pp. 397-406. 